\title{
Online TI-RADS Calculator
}

\author{
Jimmy Tanche Wang1 ${ }^{*}$, Tasha Ellchuk ${ }^{1}$, Rob Otani' ${ }^{1}$, Gary Groot ${ }^{2}$, Paul Babyn ${ }^{1}$ \\ ${ }^{1}$ Department of Radiology, Faculty of Medicine, University of Saskatchewan, Royal University of Hospital, \\ Saskatoon, Canada \\ ${ }^{2}$ Department of Surgery, Faculty of Medicine, University of Saskatchewan, Royal University of Hospital, \\ Saskatoon, Canada \\ Email: ^tanche.wang@usask.ca
}

How to cite this paper: Wang, J.T., Ellchuk, T., Otani, R., Groot, G. and Babyn, P. (2018) Online TI-RADS Calculator. Open Journal of Radiology, 8, 175-180. https://doi.org/10.4236/ojrad.2018.83020

Received: August 1, 2018

Accepted: September 3, 2018

Published: September 6, 2018

Copyright $\odot 2018$ by authors and Scientific Research Publishing Inc. This work is licensed under the Creative Commons Attribution International License (CC BY 4.0).

http://creativecommons.org/licenses/by/4.0/

\begin{abstract}
Background: Thyroid nodules are common and fine needle aspiration (FNA) or surgery is used to assess for malignancy. Thyroid Imaging, Reporting and Data System (TI-RADS) use ultrasound for non-invasive risk stratification of thyroid nodules and reduce unnecessary biopsies. This project used an online calculator and education to facilitate the application of TI-RADS in clinical practice. Methods: Retrospective review defined the baseline reporting of thyroid nodule ultrasound features. Web-based resource and presentation were used to integrate TI-RADS in reporting thyroid ultrasounds and measure the improvements in comprehensive reporting of thyroid nodules and guiding management. Results: The percentage of thyroid ultrasound reporting using TI-RADS within six months increased from $0 \%$ to $27 \%$ during the project period. Reports with TI-RADS provided twice as many recommendations compared to reports without TI-RADS. Conclusion: Online TI-RADS calculator and education have successfully facilitated the integration of TI-RADS in thyroid ultrasound reporting to provide more accurate and comprehensive reports and guide management.
\end{abstract}

\section{Keywords}

TI-RADS, Thyroid, Ultrasound

\section{Background}

Thyroid nodules are common, with a prevalence of up to $68 \%$ of adults on ultrasound [1]. Fine needle aspiration (FNA) is the most effective test in determining if a thyroid nodule is malignant and occasionally surgery is required to achieve a definitive diagnosis. But most thyroid nodules are benign and not all nodules require FNA or surgery. Over diagnosis of thyroid cancer results in many detected thyroid cancers without affecting mortality between $45 \%$ to $80 \%$ 
of cases. Recent attention has been focused on developing a non-invasive system, called Thyroid Imaging, Reporting and Data System (TI-RADS), with the use of ultrasound for risk stratification of thyroid nodules to identify clinically significant malignancies while reducing the number of biopsies performed on benign nodules.

The American College of Radiology (ACR) released a white paper in 2017 on the use of the TI-RADS. TI-RADS is based on ACR recommended standardized terms for ultrasound reporting of thyroid nodules [2]. Selected ultrasound features of thyroid nodules are combined into a score to identify nodules that warrant biopsy or sonographic follow-up. The use of TI-RADS to risk stratify incidental nodules may result in fewer unnecessary biopsies [3]. In order to facilitate the use of TI-RADS, an online calculator was developed. To serve as an educational and clinical tool, images demonstrating each of the ultrasound features are included in the online calculator.

Using an online calculator (www.TIRADSCalculator.com) for Thyroid Imaging, Reporting and Data System (TI-RADS) with images and descriptions of each of the ultrasound features as a clinical and educational tool is to guide management of incidental thyroid nodules.

\section{Method}

\subsection{Baseline Measurement}

Our institutional ethics review board approved this retrospective study and waived the requirement to obtain informed consent. All patients with thyroid sonography performed in the Saskatoon Health Region in January 2012 to May 2014 were included in this study. A retrospective case review was performed in 138 patients with 290 thyroid nodules detected on thyroid ultrasound. The study included 122 patients that were females and 16 were males with patients ranging in age from 21 to 89 years (mean, 53.1 years). None of the thyroid ultrasound reports contained a TI-RADS score. Reviewing the thyroid ultrasound reports for reporting of thyroid nodule ultrasound characteristics included in TI-RADS (composition, echogenicity, shape, margin, and echogenic foci), as well as size and location resulted in a completion rate of $43 \%$ (Figure 1).

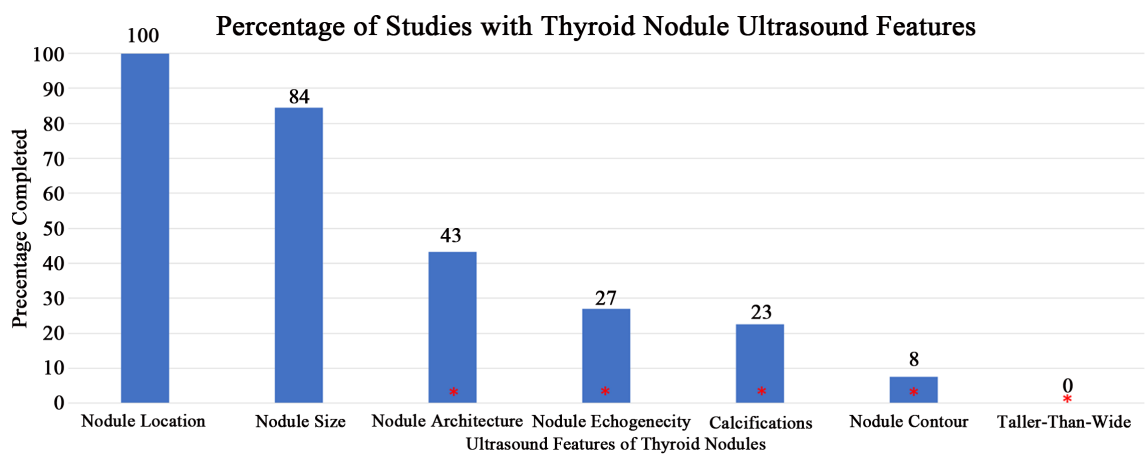

Figure 1. The reporting rate of thyroid nodule ultrasound features prior to the intervention. *Thyroid nodule ultrasound features in TI-RADS. 


\subsection{Design}

An online calculator (www.TIRADSCalculator.com) for Thyroid Imaging, Reporting and Data System (TI-RADS) with images and descriptions of each of the ultrasound features was developed (Figure 2 and Figure 3). The website was disseminated to radiologists in Saskatoon via e-mail. A grand round presentation was provided by Dr. Otani on TI-RADS and the online calculator.

Six months later, thyroid ultrasound studies predominantly within the first week of December 2017 were reviewed. A total of 92 studies were included with an average age of 56 years old. The proportion of females to males was 3:1.

\section{Results}

Twenty-seven percent of the studies reviewed reported a TI-RADS score. The reports that contained a TI-RADS scored provided a recommendation 2.2 times

\section{TI-RADS Calculator}

Online calculator for Thyroid Imaging Reporting and Data System (TI-RADS) based on 2017 ACR white paper with guidance on fine needle aspiration (FNA) and follow-up.

Click here for images and description for each of the ultrasound features

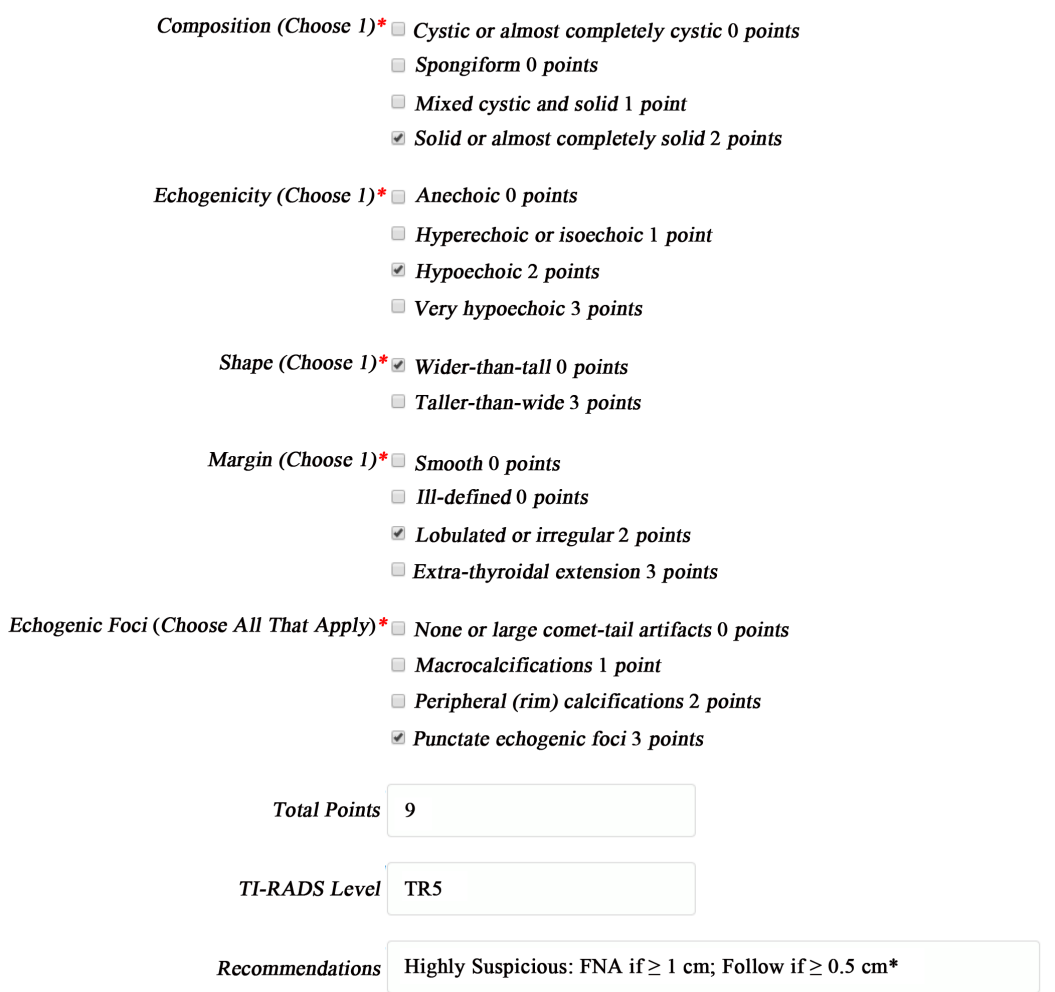

\section{Reset}

Figure 2. Screen capture of the online TI-RADS calculator (www.TIRADSCalculator.com). 


\section{Echogenic Foci}

Large comet tail artifacts

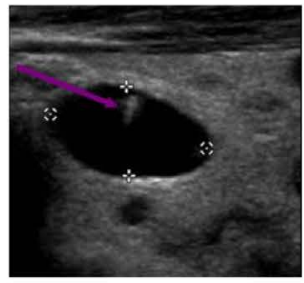

- Comet-tail artifacts: Deeper echoes attenuated with decreased width resulting in a triangular shape. V-shaped, $>1 \mathrm{~mm}$, in cystic components.

Macrocalcifications

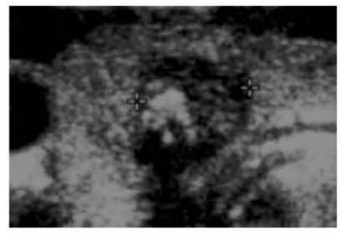

- Macrocalcifications: Calcifications with posterior acoustic shadowing

Peripheral rim calcifications

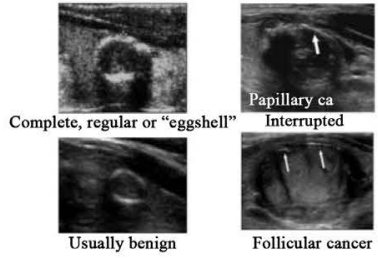

- Peripheral calcifications: Calcifications at periphery of the nodule. Complete or incomplete along margin.

Punctate echogenic foci

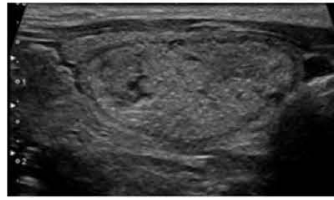

- Punctate echogenic foci: $<1 \mathrm{~mm}$ with no posterior acoustic shadowing. May have small comet-tail artifacts.

Figure 3. Screen capture of an example of the images and description for each of the thyroid nodule ultrasound features in TI-RADS.

more than reports that did not contain a TI-RADS score. Reviewing the thyroid ultrasound reports without TI-RADS scores for reporting of thyroid nodule ultrasound characteristics included in TI-RADS (composition, echogenicity, shape, margin, and echogenic foci), as well as size and location resulted in a completion rate of $39 \%$ (Figure 4). 


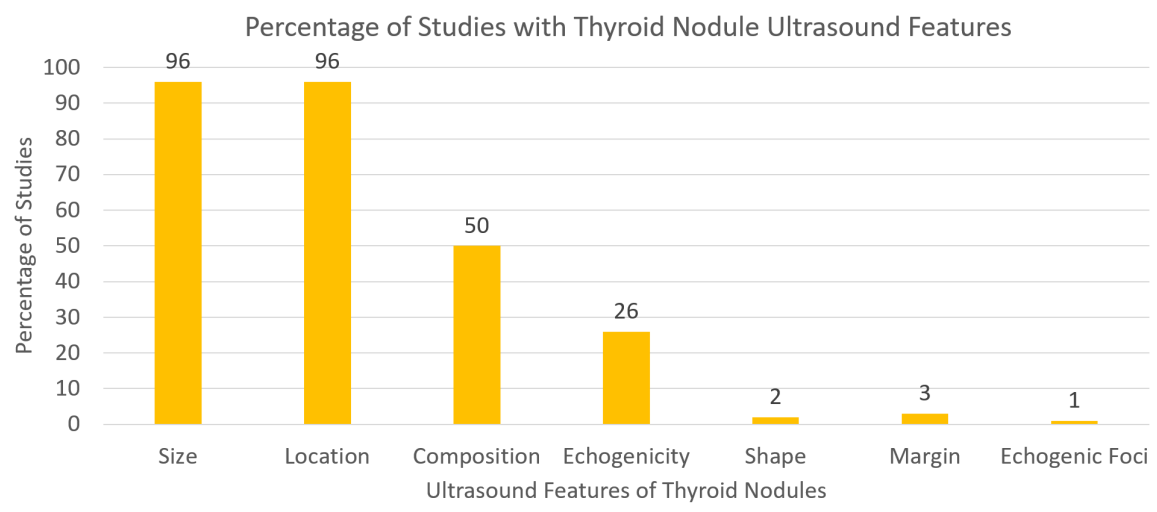

Figure 4. The reporting rate of thyroid nodule ultrasound features in reports without TI-RADS scores post-intervention.

\section{Discussion}

The uptake of TI-RADS would be expected to be highest shortly after the presentation but continued usage of TI-RADS would subsequently decrease with time. To best assess for sustainable use of TI-RADS, a longer term for assessment of six months was selected. The assessment of the uptake of TI-RADS six months after the presentation is thus a conservative estimate of the use of TI-RADs post-intervention.

The reporting rate of thyroid nodule ultrasound characteristics were similar in thyroid ultrasound reports without TI-RADS scores after the presentation compared to reports prior the presentation. This finding serves to validate the results.

\section{Conclusion}

An online calculator was developed for TI-RADS based on the ACR white paper in 2017 to facilitate the application of TI-RADS in clinical practice. Images and description of each of the ultrasound features of thyroid nodules are included to serve as an educational and clinical tool on the use of TI-RADS. Using TI-RADS in ultrasound based risk stratification of incidental thyroid nodules will guide management and potentially reduce unnecessary thyroid biopsies and interventions.

\section{Declaration of Conflicting Interests}

The authors declared no potential conflicts of interest with respect to the research, authorship, and/or publication of this article.

\section{Funding}

The authors received no financial support for the research, authorship, and/or publication of this article.

\section{References}

[1] Tessler, F.N., Middleton, W.D., Grant, E.G., Hoang, J.K., Berland, L.L., Teefey, S.A., 
et al. (2017) ACR Thyroid Imaging, Reporting and Data System (TI-RADS): White Paper of the ACR TI-RADS Committee. Journal of the American College of Radiology, 14, 587-595. https://doi.org/10.1016/j.jacr.2017.01.046

[2] Grant, E.G., Tessler, F.N., Hoang, J.K., Langer, J.E., Beland, M.D., Berland, L.L., et al. (2015) Thyroid Ultrasound Reporting Lexicon: White Paper of the ACR Thyroid Imaging, Reporting and Data System (TIRADS) Committee. Journal of the American College of Radiology, 12, 1272-1279. https://doi.org/10.1016/j.jacr.2015.07.011

[3] Wang, J.T., Babyn, P., Groot, G. and Otani, R. (2016) Electronic Synoptic Reporting of Thyroid Nodules: Potential for Reduction in Number of Patients Undergoing Thyroid Nodule Biopsies. Open Journal of Radiology, 6, 233-242.

https://doi.org/10.4236/ojrad.2016.63031 